
cultures

Les cahiers de l'Acedle

10-2 | 2013

Apprendre les langues autrement

\title{
Approche culturelle et écriture réflexive pour un public Lansad
}

Isabelle Salengros-Iguenane

\section{OpenEdition}

Journals

Édition électronique

URL : http://journals.openedition.org/rdlc/1624

DOI : $10.4000 /$ rdlc. 1624

ISSN : 1958-5772

Éditeur

ACEDLE

Référence électronique

Isabelle Salengros-Iguenane, "Approche culturelle et écriture réflexive pour un public Lansad »,

Recherches en didactique des langues et des cultures [En ligne], 10-2 | 2013, mis en ligne le 07 juin 2013, consulté le 23 avril 2019. URL : http://journals.openedition.org/rdlc/1624 ; DOI : 10.4000/rdlc. 1624

Ce document a été généré automatiquement le 23 avril 2019

\section{(c) $($ ) $(9)$}

Recherches en didactique des langues et des cultures is licensed under a Creative Commons AttributionNonCommercial-NoDerivatives 4.0 International License 


\title{
Approche culturelle et écriture réflexive pour un public Lansad
}

\author{
Isabelle Salengros-Iguenane
}

Je remercie Françoise Demaizière pour sa relecture et ses commentaires.

\section{Introduction}

1 Notre recherche se déroule à l'École des Ponts ParisTech, une grande école d'ingénieurs, qui accueille chaque année une centaine d'étudiants internationaux. Ils suivent une formation scientifique (formation d'ingénieurs - cycle Master) et réalisent une mobilité pouvant aller d'un semestre à deux ans.

2 Nous avons précédemment mis en évidence les difficultés, d'ordre socioculturel, des étudiants en mobilité à l'école (Salengros-Iguenane, 2010a) et construit un module de formation en français langue étrangère (FLE) intégrant Internet comme support pour la pédagogie. Son objectif est d'aider les étudiants à mieux comprendre les particularités "culturelles" de la communauté française de l'école et à mieux s'y insérer d'où son titre Partager les cultures des Français.

3 Notre recherche s'appuie sur les particularités de la formation de jeunes adultes et nous nous intéressons à l'importance de la responsabilisation et de l'autonomie. Dans cette optique les apprenants ont été invités à produire un journal de bord permettant de mettre en mots leur vécu et leurs réflexions socioculturelles. Le journal de bord, dans le cas de notre étude, regroupe un échantillon de productions de l'apprenant qui illustrent sa réflexion personnelle et ses différentes recherches ainsi que l'état de ses connaissances et de ses compétences.

4 La formation traite de certains éléments socioculturels partagés, des lieux communs qui circulent et qui semblent indispensables pour s'intégrer dans la communauté de l'école (thématiques culturelles telles que le cinéma, la chanson, la BD). Nous nous sommes tournée vers la sphère de la culture dite anthropologique ou partagée (Galisson, 1991 : 116-117; Galisson \& André, 1998 : 6-7 ; Pothier, 2003 : 22-28) tout en considérant celle-ci 
avec prudence afin d'éviter une vision fossilisée ou figée de la culture, que cette conception pourrait impliquer. Nous nous sommes également appuyée sur certaines préconisations propres aux recherches sur l'interculturel, l'approche herméneutique par exemple (Huver \& Springer, $2011: 265$; Abdallah-Pretceille, $2003: 15$ ). Des entrevues de groupes et des questionnaires (recueil de données) ont permis d'aboutir à des profils d'élèves (voir Salengros-Iguenane, 2010a) et ont aidé à l'élaboration du module.

Dans cet article, nous rappellerons, tout d'abord, les principes didactiques et méthodologiques que nous avons retenus s'agissant du journal de bord. Nous analyserons ensuite les données recueillies dans ces journaux et mettrons en valeur en particulier qu'il est le témoin du cheminement personnel et d'une certaine relativisation culturelle chez les apprenants.

\section{Les adultes et l'apprentissage}

6 La littérature de recherche montre qu'un public d'adultes apprécie les activités qui impliquent de la complexité, des défis, de l'autonomie et une certaine responsabilisation (Malglaive, 1990 : 116; Martin \& Savary, 2004: 47 ; Springer, 1996:6-11). Les activités proposées doivent mobiliser des opérations de haut niveau, par exemple des productions langagières "ouvertes", telles que la note de synthèse, le compte rendu oral ou écrit (Guichon, 2006:17-18), activités peu balisées, laissant une certaine liberté, confrontant à des obstacles, des questionnements et plaçant en position de responsabilité. Le journal de bord semble un écrit approprié pour notre public car il peut être un moyen de mettre en place ce type d'activités.

\section{Approche didactique et journal de bord}

Plusieurs appellations existent (carnet de bord, journal de formation, journal de bord, "portfolio", "dossier", journal réflexif, journal d'apprentissage) pour un objet qui contient les réflexions de l'apprenant (à propos de son apprentissage) et ses productions (travaux réalisés et profitables pour sa formation) et dont la forme diffère parfois. Nous parlons quant à nous de "journal de bord".

Dans le cadre de la formation professionnelle, le journal de bord est, en général, composé de deux parties (une partie analytique et une partie "dossier"). La partie analytique du journal est le témoin et l'outil du développement de l'apprenant, de sa prise de conscience, de sa métamorphose (voir les travaux de Causa, 2007 ; Chiss, 2007 ; Guichon, 2012: 201; Molinié, 2011: 61; Tardif, 1998:79). Le journal réflexif utilisé pour l'apprentissage des langues permet, quant à lui, de développer la mise en place d'une attitude réflexive: prise de distance sur son apprentissage, prise de conscience de sa progression dans son apprentissage dans la matière (Cadet, 2006).

9 Le journal de bord que nous avons proposé a été conçu en fonction de notre public et possède des spécificités par rapport à celui documenté dans les recherches. Les modifications sont délibérées : adaptation de l'outil à notre projet, au contenu du module, au public visé et aux exigences institutionnelles. Le journal de bord demandé n'est pas composé, comme dans le journal de formation, d'une partie analytique et d'une partie "dossier". La réflexion de l'étudiant est contenue dans les productions ou bien sous forme 
de paragraphes réflexifs à la suite de la production. Il ne contient pas de biographie langagière ni de passeport des langues.

Nous avons souhaité recourir à un journal de bord pour que l'apprenant mette en place une réflexion personnelle, un questionnement sur soi et sur autrui (Vezina, 2002: 9; Molinié, 2011 : 29) dans l'optique du module. Le journal de bord peut permettre de rendre compte du cheminement personnel de l'apprenant et lui donner l'occasion d'exprimer ses intentions (Vézina, 2002: 10), ce qui nous a semblé tout à fait indiqué par rapport aux objectifs du module et pour l'approche culturelle retenue. Il peut aider à la mise en place d'une réflexion socioculturelle et à une conscientisation des questions portant sur "la traversée de l'espace social" (Molinié, 2007) comme source d'une expérience anthropologique. Utilisé comme journal de recherche, il peut être un moyen de rendre compte des découvertes que réalise l'apprenant tout en prévoyant une posture de recherche et un apprentissage langagier (Scheepers, 2006). Dans le meilleur des cas, la réflexivité, que le journal de bord engendre, conduit les apprenants à prendre conscience de la dimension interculturelle de leur expérience (Molinié, 2011 : 77) et à "élaborer des hypothèses qui prennent en compte différentes variables situationnelles (contexte relationnel, statut des interactants, rapports de pouvoirs, etc.) et structurelles (économie, politique, histoire, etc.)" (Huver \& Springer, 2011: 265). Dans ce cas, il peut offrir l'occasion de mettre en place, dans une certaine mesure, les principaux objectifs des rencontres interculturelles "renouvelées" suggérées par F. Dervin (2011 : 124-125).

11 L'introduction d'un journal de bord peut être une tâche complexe (Guichon, 2012 : 202 ; Molinié, $2011: 64$; Rosen, 2006 : 71). L'accueil réservé à ce type de travail par les étudiants n'est pas toujours favorable, nous l'avons constaté. Nous avons parfois dû faire face aux réticences et à l'incompréhension de certains apprenants compte tenu du fait que ce type de travail n'est pas courant dans leur parcours académique.

\section{Le journal de bord et sa mise en œuvre}

\section{Le module}

12 Le groupe d'apprenants est généralement composé d'une dizaine de personnes de nationalités hétérogènes d'un niveau A2-B1 du CECRL (Conseil de l'Europe, 2001). Le module a pour objectif, d'une part, d'aider les participants à s'approprier des éléments de la culture cible (et plus particulièrement à s'adapter au cursus d'une grande école), et d'autre part, de permettre de "provoquer" des échanges voire des connivences avec les membres de la communauté française de l'école (voir Salengros-Iguenane, 2010b: 278-297). Les apprenants travaillent sur différentes thématiques (le cinéma, la chanson, la $\mathrm{BD}$, la presse, l'histoire, etc.) et réalisent des recherches encadrées, à l'aide de fiches, sur Internet. Il existe deux types de fiches : les fiches de travail contenant les consignes des activités et les fiches de recherche contenant la liste de sites Internet sélectionnés pour chaque thème (voir Salengros-Iguenane, 2010b: 292). Nous avons adopté, en tant qu'enseignante, une posture non directive afin de favoriser le travail collectif et la construction des connaissances. 


\section{Le journal de bord}

13 Ce travail, réalisé individuellement, est rendu deux fois dans le semestre : à mi-parcours et en fin de parcours. Le journal demandé rejoint les points évoqués précédemment: prise de distance, prise de conscience, témoin du développement de l'apprenant, mise en mots du vécu, réflexion socioculturelle, apprentissage par l'exploration. Il vise également à mettre en place les éléments suivants.

- Réaliser des recherches personnelles tel un « archéologue ». L'apprenant doit essayer ainsi d'en apprendre davantage sur les thèmes abordés au cours des séances et de "reconstruire, avec les éléments dont [il] [dispose] et avec autant d'authenticité que possible, le puzzle que constitue l'autre culture" (Furstenberg \& English, 2006). C'est également l'occasion "d'approfondir leur réflexion au sujet des expériences qu'ils vivent tant sur le plan cognitif et affectif que relationnel et d'effectuer des prises de conscience personnelles" (Molinié \& Leray, 2002).

- Prolonger ce qui a été découvert lors des séances en utilisant les liens hypertextes proposés sur les fiches de travail. Grâce aux sites Internet "bruts" (non pédagogiques) l'apprenant accède ainsi à différentes vidéos, articles de presse, documents audio, etc. qu'il n'aura peutêtre pas eu le temps de consulter en présentiel.

- Permettre, nous l'espérons, grâce aux recherches réalisées pour le journal de bord et à la réflexion que celles-ci induisent, que l'apprenant ne :

"[survalorise] pas 'la culture', définie comme un système qui détermine les individus, mais qu'[il l'envisage] comme un élément parmi d'autres des interactions" et qu'il "soit en mesure de construire une pluralité d'hypothèses en mobilisant différents paramètres explicatifs (psychologiques, sociologiques, politiques, culturelles), de manière à relativiser, voire à déconstuire, le rôle de 'la' culture (notamment lorsqu'elle est uniquement définie relativement au critère de l'appartenance nationale) " (Huver \& Springer, $2011: 267)$.

Le journal de bord regroupe ici un échantillon de productions de l'apprenant, réalisées en dehors des séances, qui permettent d'accéder à sa réflexion personnelle et qui exposent ses différentes recherches. L'apprenant est libre d'adopter une présentation thématique ou chronologique ou toute autre présentation. Il est invité à mentionner, dès l'introduction de son travail, les raisons pour lesquelles il s'est engagé dans ce module et ce qu'il en attend. Cette information permet, par exemple, d'ajuster le module s'il y a lieu.

Consignes pour le journal de bord.

Il s'agit d'un journal ${ }^{1}$ que vous remplirez au fur et à mesure de vos recherches. Il va vous accompagner tout au long de la découverte des thèmes de Partager les cultures des Français.

C'est un travail individuel. Il sera à l'image d'un carnet de bord (papier ou numérique), d'un journal d'anthropologue (étude de la diversité) ou d'ethnologue (étude de la différence), etc. dans lequel vous indiquerez par exemple: vos découvertes, vos questions, vos réflexions (chocs, surprises agréables ou désagréables), des articles de presse, des interviews d'élèves, des documents récupérés pendant le semestre dans l'École, le métro, etc. L'enseignant vous proposera également des activités (pour approfondir chaque thème). Celles-ci peuvent être insérées dans le journal de bord. Les productions sont personnelles, il ne faudra pas recopier des propos qui ne sont pas les vôtres. Si vous vous appuyez sur les propos d'une personne, il faudra indiquer les titres des ouvrages, les sites Internet consultés ou l'émission visionnée, etc. 


\section{Analyse et discussion} qui est vécu et interprétation, qui stimulent la prise de distance). Les apprenants évoquent ce qu'ils ont découvert, "appris". Ils ont parfois réussi à laisser libre cours à leur pensée ce qui nous a semblé positif: cette écriture réflexive semble retracer le cheminement personnel, les divers questionnements par lesquels ils passent, le développement de la capacité de prise de distance des apprenants.

En cinq jours je rentre chez moi. Ce samedi je retrouverai ma famille à l'aéroport et mes amis aux pubs. Ils m'ont manqué beaucoup pendant ces quatre moins. Néanmoins, je n'avais jamais profité de seize semaines comme celles-ci. Seize semaines pendant lesquelles j'ai connu le plus grand nombre de personnes en même temps, chacune d'une nationalité différente, avec un accent différent, dès le Brésil au Viêt-Nam, dès le Maroc à la Roumanie. Seize semaines pendant lesquelles j'ai découvert les méthodes d'enseignement et le fonctionnement d'une grande école, où nous sommes traités de manière bien différente par rapport à nos écoles d'origine. Seize semaines complètes pour parcourir Paris, quartier par quartier, monument à monument, jour à jour, même si Paris reste toujours interminable.

Deux apprenantes mettent en place un mécanisme de conscientisation vis-à-vis du rôle des idées véhiculées par certains films, visions stéréotypées, aseptisées, à caractère situé.

Je crois que Le fabuleux destin d'Amélie Poulain est un très bon film, de la même façon que la bande sonore, je les ai trouvés très intéressantes. J'ai lu qu'il y a eu des critiques qui ont attaqué pour être peu réaliste, parce que dans le film on ne représente des minorités africains qui habitent près de l'héroïne, et car Amélie est une serveuse qui aujourd'hui dans le monde réel n'aurait pas assez d'argent pour habiter où elle a son appartement. Mais, tout de même, je l'ai beaucoup aimé.

Pendant tout l'histoire on ne voit pas une seule fois quelque monument ou image clichée de Paris. J'ai trouvé ceci très intéressant. Et après réfléchir j'ai arrivé à la conclusion que la vie en Paris c'est comme ça, on ne fait pas attention à cela qu'est en tour de nous.

Les remarques d'une autre apprenante concernant la presse (manière dont sont traités les sujets) ou bien le cinéma (hermétisme de certains films) montrent un certain positionnement réflexif vis-à-vis de ce qu'elle voit, observe, entend et lit. 
En autres, les presses quotidiennes traite de la culture du pays et cela est différent, la même nouvelle est écrite des beaucoup des façons pour s'adapter et être plus attractives aux citoyens [...] En fait, chaque société invente ses propres expériences et les choses qui sont divertissement dans un pays peut être ridicule en autre.

19 Quelques indices montrent que des apprenants ont adopté une certaine prise de distance vis-à-vis de la "culture" cible voire une relativisation culturelle. Nous pouvons espérer que cette posture est liée à l'étude des thématiques culturelles proposée.

Un participant mentionne l'intérêt d'étudier des éléments socioculturels pour comprendre l'autre.

Je pense que la connaissance de la culture est une chose indispensable pour bien apprendre une langue. Parce que, à mon avis, ça n'est pas seulement apprendre à bien parler, il faut apprendre et comprendre la base de tout, la culture qui fait des expressions être d'une manière et non d'autre, c'est la culturel d'un pays qui nous fait parler d'une forme et non d'autre. Si quelqu'un bien comprendre la culture, c'est sûr qu'un jour il pourra parler avec des autres mais sinon à mon avis, il pourra seulement mettre la langue dans le CV, il ne sauvera pas parler avec de quoi parler avec des autres et ne sauvera pas comprendre des autres...

21 Une étudiante a souhaité ne pas se limiter aux éléments socioculturels français et a voulu également "partager la culture des Français d'origine chinoise". Elle présente alors sa journée et ses découvertes autour du nouvel an chinois à Paris.

Le dimanche 10 février on a pu jouir des fêtes de célébration du nouvel en chinois qui ont eu lieu au 13ème arrondissement. [...] les chinois du 13ème arrondissement n'ont pas voulu perdre l'opportunité de nous montrer leurs habitudes traditionnelles et leurs danses typiques.

Des participants évoquent l'existence d'une barrière culturelle qui semble possible à surmonter. Ils mentionnent également le fait que la proximité géographique ne signifie pas toujours ressemblance. L'accès à certains documents étudiés pour le module et les thèmes permettent parfois de favoriser la mise en place d'une relativisation culturelle. Voici deux commentaires.

(1) Je pensais qu'il serait plus facile de parler avec des gens d'une autre nationalité, mais peut-être j'étais trompée. J'ai un ami avec lequel j'ai parlé sur ce sujet, et il me dit qu'il pense qu'il existe une barrière culturelle. Je crois que c'est possible de la surmonter, mais quand même, il est difficile! Même entre des nationalités très proches comme peuvent être la française et l'espagnole, ou l'italienne et l'espagnole, il y a des différences.

(2) Après lire le roman graphique je peux dire que je comprends mes amis iraniens. En fait je crois que je comprends mieux tous mes nouveaux amis. J'explique : pendant mon actuelle expérience en France j'ai connu beaucoup des gens de différentes origines. Je dois confesser qu'au début j'ai trouvé plusieurs de mes nouveaux amis un peu bizarres... Mais avec le temps, $\mathrm{j}$ 'avais constaté que la différente culture, les différentes origines influencent beaucoup notre comportement. propos. Elle transmet ses émotions, son désarroi parfois mais également ce qui est positif pour elle, qu'elle intègre et qui lui permet de construire son cheminement. Son journal de 
bord est le témoin du mécanisme de revalorisation du regard qu'elle porte sur son pays. Il semble que cela ait été possible, non seulement grâce à ce qu'elle a vécu au fur et à mesure de son séjour mais également grâce à l'étude des documents et des thèmes abordés au cours du module. Le journal de bord permet, nous l'espérons, de contribuer à ce cheminement par la mise en mots qu'il demande. Les éléments étudiés, le travail demandé pour le journal de bord, ses réflexions et sa prise de distance vis-à-vis de ce qu'elle observe lui permettent de poser un autre regard sur sa propre société.

En Espagne, nous croyons que presque toutes les pays européens sont plus développés que le nôtre, et en fait j'ai toujours eu cette opinion. Mais après avoir habité ces trois mois ici, j'ai découvert que cela n'est pas vrai !

Un autre participant change également de point de vue sur certains éléments de son pays. Il analyse ce qu'il observe, à distance, de son pays sous forme de rapprochement avec ce qui l'entoure. Il souligne d'ailleurs l'intérêt de l'activité proposée (observer la une d'un journal français et celle d'un journal de son pays pendant une semaine).

Cette séance a été curieuse car j'ai l'opportunité de regarder la presse quotidienne de la France et comparer avec la presse du Portugal. Cela a été bon parce que m'a permis de constater qui la presse à chez moi n'est pas mal $\mathrm{du}$ tout. Je veux dire avec ceci qui se semble beaucoup avec la presse française. [...] j'ai trouvé l'exercice intéressant, parce qu'il m'a fait réfléchir sur la mentalité dans mon pays.

Les apprenants se laissent parfois aller à des généralisations. Le processus de relativisation culturelle était encore instable au moment de la rédaction des journaux de bord. Une apprenante, par exemple, est consciente que certains stéréotypes sont faux ou que certaines généralisations concernant son pays sont vraiment exagérées. Elle prend cependant conscience qu'il est facile de généraliser abusivement pour se différencier des autres et affirmer son identité face à l'autre.

J'ai trouvé cette bande dessinée très drôle car elle surtout satirique, même si, à mon avis, il y a quelques moments où elle est assez exagérée. Elle touche au sujet comme la façon d'être d'un peuple et ses idées politiques. En chaque pays, il y a des différentes régions et on a des différents stéréotypes pour chacune d'elles. Mais... sont-ils vrais? [...] En Espagne, je viens d'une des trois régions avec une langue propre, une région où le nationalisme est assez important, et d'ailleurs, les galiciens, nous sommes aussi stéréotypés. J'ai demandé à quelques amis leur opinion que le reste des espagnols ont sur nous [...]. Donc je crois que les stéréotypes sont toujours des exagérations, mais au fond, il y a quelque vrai.

Dans certains cas, la généralisation peut aller jusqu'à la dévalorisation du regard que l'apprenant porte sur son pays d'origine.

Je trouve la presse française de meilleure qualité, les journaux brésiliens parlent beaucoup sur le foot, sur les programmes de TV, mais ils ne parlent pas les nouvelles nationales vraiment importantes sur les décisions politiques, etc.

Particulièrement, en discutant sur politique avec mes amies français, j'ai pu observer qui ici en France le candidat ne gagne pas tout seule le poste de président, évidemment ça dépend de son charisme et compétence, mais le parti politique, l'organisation de ce parti, la visée et les autres personnes qui iront également occuper des hautes poste du gouvernement, comptent 
beaucoup, au contraire du Brésil, où le candidat est le plus important et peu importe qui va travailler avec lui. Bien sûr, c'est une opinion personnel, mais à grosso modo, je trouve que à cause d'un mauvaise formation (notamment l'éducation en France a atteint un niveau plus élevé par rapport au Brésil), le Brésilien moyen n'aime pas la politique et ne sait pas que un gouvernement ne peut pas être conçue pour une unique personne.

Le journal de bord est également le témoin de l'adaptation de l'apprenant à l'environnement pendant la période de mobilité. Une étudiante est étonnée, surprise, au retour dans son pays pour les vacances, par ce qu'elle voit, entend, observe alors qu'elle ne s'est absentée que deux mois. Les étudiants étrangers doivent s'adapter à la vie dans le pays d'accueil, mais également se réadapter à la vie dans leur pays d'origine (MurphyLejeune, 2005 : 136-137).

J'ai pris ma valise et enfin j'ai rencontré mes parents. Ils avaient très envie de me voir, et moi de les voir aussi, mais c'était très bizarre pour moi être en Espagne une autre fois : les gens parlaient espagnol, tout était plus calme, l'aéroport était plus petite... mais je crois que j'ai eu cette impression car j'avais été beaucoup de temps dehors!

\section{Le journal de bord : le vécu des apprenants}

Quatre apprenants transmettent des informations sur leurs habitudes, leur ressenti, leurs stratégies, leurs surprises vis-à-vis des thématiques étudiées et se livrent même personnellement. La présence de ces propos nous semble positive. On y perçoit, à notre sens, la mise en place d'une prise de distance, l'apprentissage d'une posture réflexive qui donne de l'épaisseur à ce qui est relevé. Ces apprenants semblent s'impliquer réellement dans les activités proposées. En n'hésitant pas à se dévoiler, ils montrent qu'ils se sentent en confiance dans le cadre proposé, ce qui ne peut que favoriser l'apprentissage.

Quand j'ai plus de temps (les dimanches), je préfère lire "El Paìs". C'est le journal le plus lu de l'Espagne. Il est plutôt à gauche. Moi, j'ai l'habitude de le lire à l'inverse, c'est-à-dire, je commence par la dernière page, pour avoir les programmations de la télé et les sports. Puis je continue avec les dessins et les lettres des lecteurs au directeur, et finalement, si j'ai encore des forces, je lis la politique nationale et internationale.

Cependant, c'est quelque chose que m'a toujours plu, le fait de feuilleter un quotidien à la cafétéria, où chez un ami, par exemple, en lisant seulement les gros titres et les chapeaux. Au contraire, la radio c'est un moyen de communication que j'utilise souvent [...] Je préfére écouter les conversations des journalistes, ils me tiennent plus compagnie.

Deux étudiants s'investissent émotionnellement dans leurs commentaires. L'un d'entre eux a d'ailleurs mis en place une réflexion concernant certaines œuvres, certains sujets tout en évoquant ses émotions.

J'ai un problème. J'associe les chansons au moment auquel je les écoute. C'est un vrai problème. Le plus j'aime la chanson, le plus je le lie au lieu, à la situation et à l'époque où je l'ai appris. Et puis, je ne peux plus l'écouter après, la nostalgie ne me le permet pas. (À propos du thème de la chanson.)

Chaque histoire plus malheureuse que la précédente, chaque voix plus captivante. Elles atteignent à transmettre la solitude, le déracinement et la 
peine d'une « copla » espagnole et d'un tango argentin. C'est magnifique. Je les écoute encore et toujours. Jusqu'au moment auquel je ne pourrais plus. qu'ils puissent s'habituer à ce qu'ils trouvaient auparavant surprenant. Ils font également part de quelques regrets (face à cette situation par exemple), de leurs angoisses.

Une chose est claire : ces élections sont gagnées par le plus offrant. Personne ne demande quels sont les programmes des groupes; eux-mêmes, ils ne présentent pas des contenus détaillés. Le vote dépend absolument de la campagne électorale, de qui prépare la meilleure présentation, la plus drôle, de qui donne lus de prix [...]. Cela est très étonnant la première fois, mais à la fin de la semaine, c'est quelque chose de totalement normal. C'est amusant de se laisser acheter d'une manière si évite, bien qu'il soit aussi un peu triste. (À propos de la campagne électorale du bureau des élèves.)

De plus on doit démarrer la recherche de stage pour l'été, choisir les domaines qui nous intéressent, rédiger les lettres de motivation, cacher les nerfs aux entretiens, et se préoccuper de l'achat des vêtements appropriés, cela compte aussi !

31 Un apprenant décrit sa surprise quant à la composition du gouvernement de François Fillon. Un autre s'étonne des mouvements sociaux.

Moi je ne savais pas qu'il y avait des ministres de gauche dans un gouvernement de droite. C'est bizarre!

En France une des choses plus bizarres pour moi lors de mon séjour ici a été le numéro des grèves et manifestations qui sont fait. Aujourd'hui Le monde détache trois articles à ce type d'événements.

Certains apprenants réalisant une mobilité à l'étranger semblent mûrir rapidement et être solides face à ce qu'ils vivent. Un étudiant souligne

On aura des problèmes, on mettra du temps pour s'habituer à la nouvelle situation ... mais à la fin, on y réussira. J'en suis sûre.

Ce recueil d'informations nous permet de dénouer certaines incompréhensions ou bien de lutter contre certaines généralisations. Le journal de bord est un moyen de recueillir des données afin d'essayer de mieux répondre, lors des séances qui suivront sa lecture, au plus près aux besoins, aux interrogations, aux attentes des apprenants. Le journal de bord révèle la manière dont les apprenants voient ou interprètent les informations étudiées. Il est important, dans ce cas de figure, de sensibiliser les participants à la complexité de l'identité et du terme de "culture" pour éviter si possible de trouver, dans leurs écrits, des généralisations excessives (sous forme de commentaire en marge du journal de bord ou bien en consacrant des moments pendant les séances aux "dangers" de la généralisation, du jugement à partir d'une infime partie émergée de la "culture" d'attache ou de la "culture" cible). 


\section{Intérêt du journal de bord pour les participants, étudiants et enseignant} connaissance de l'espérons, en partie appropriées, tout au long du semestre et, d'autre part, d'observer comment l'apprenant a éventuellement évolué au cours de la formation. Il permet de repérer, grâce aux remarques présentes dans le texte, si une évolution a eu lieu en termes de codes culturels entre les participants et les élèves de l'école (voir Salengros-Iguenane, 2010b : 331-340). Voici trois commentaires suggérant de telles évolutions.

J'ai réussi de comprendre quelques blagues de mes collègues français et de démontrer ma connaissance en chantant quelques extraits.

Je pense que cette connaissance $\mathrm{m}$ 'a permis et me permettra de participer activement aux conversations des français, et même comprendre certains articles des journaux ou journal télévisé.

À mon avis, tout ce qu'on a appris pendant les séances [...] m'a servi pour m'introduire dans les conversations des Français et même pour les connaître pour la première fois. Le fait que je sois capable de connaître certains chanteurs ou films, leur montre que je suis intéressée pour approfondir leur culture. Et aussi quand je reçois visite de l'Espagne, il semble que je maîtrise le quotidien parisien.

\section{Pour finir}

Nous espérons avoir montré que le journal de bord est un outil pertinent pour l'approche culturelle retenue, qui consiste à sensibiliser les apprenants à Partager les cultures des Français et à apprendre la langue, et pour le public spécifique d'une école d'ingénieurs. Cette activité a été bien accueillie, elle a permis d'impliquer les apprenants et de les mobiliser sur des tâches laissant une certaine liberté et ouvrant sur les questionnements souhaités tout en fournissant à l'enseignante des indices utiles à la conduite de l'enseignement. 
Nos résultats permettent de compléter les travaux antérieurs sur le journal de bord en mettant en valeur en particulier qu'il est le témoin du cheminement personnel et d'une relativisation culturelle des apprenants. Pour ce qui est des perspectives d'évolution, à l'instar de N. Guichon (2012: 202), nous pourrions envisager de socialiser l'écriture de ce journal en demandant à des volontaires de lire des fragments de leurs journaux devant le groupe "afin de mettre en relation l'individuel et le collectif". Dans le même ordre d'idées, nous pourrions profiter des potentialités d'une plateforme de type collaboratif pour publier ces fragments et les rendre accessibles au groupe de participants et construire des activités autour de cette publication.

\section{BIBLIOGRAPHIE}

Abdallah-Pretceille, M. (2003). Former et éduquer en contexte hétérogène. Pour un humanisme du divers . Paris : Anthropos.

Cadet, L. (2006). "Des notions opératoires en didactique des langues et des cultures : modèles ? Représentations ? Culture éducative ? Clarification terminologique". Les cahiers de l'Acedle, $\mathrm{n}^{\circ} 2$, pp. 36-51.

Causa, M. (2007). "Un outil pour apprendre à se former : le journal de formation". Causa, M. (coord.). Formation initiale en français langue étrangère : actualité et perspectives. Le français dans le monde - Recherches et applications, $n^{\circ} 41$, pp. 169-179.

Chiss, J.-L. (2007). "Didactique des langues et histoire(s) de formation(s) ". Causa, M. (coord.). Formation initiale en français langue étrangère : actualité et perspectives. Le français dans le monde Recherches et applications, $\mathrm{n}^{\circ} 41$, pp. 182-191.

Conseil de l'Europe (2001). Cadre européen commun de référence pour les langues - Apprendre, enseigner, évaluer. Paris : Didier.

Dervin, F. (2011). Impostures interculturelles. Paris : L'Harmattan.

Furstenberg, G. \& English, K. (2006). "Communication franco-américaine via Internet". DejeanThicuir, C. \& Mangenot, F. (coord.). Les échanges en ligne dans l'apprentissage et la formation. Le Français dans le monde - Recherches et applications, $\mathrm{n}^{\circ}$ 40, pp. 178-191.

Galisson, R. (1991). De la langue à la culture par les mots. Paris : CLE International.

Galisson, R. \& André, J.-C. (1998). Dictionnaire de noms de marques courant. Paris : Didier Érudition. Guichon, N. (2006). Langues et TIC - Méthodologie de conception multimédia. Paris : Ophrys.

Guichon, N. (2012). Vers l'intégration des TIC dans l'enseignement des langues. Paris : Didier.

Huver, E. \& Springer, C. (2011). L'évaluation en langues. Paris : Didier.

Malglaive, G. (1990). Enseigner à des adultes. Paris : PUF.

Martin, J.-P. \& Savary, É. (2004). Formateur d'adultes. Lyon : Chronique Sociale. 
Molinié, M. (2007). "Faire de son biculturalisme un objet de savoir en sciences humaines". Causa, M. (coord.). Formation initiale en français langue étrangère : actualité et perspectives. Le français dans le monde - Recherches et applications, $\mathrm{n}^{\circ}$ 41, pp. 147-156.

Molinié, M. (2011) (dir.). Démarches portfolio en didactique des langues et des cultures. Enjeux de formation par la recherche action. Cergy-Pontoise : CRTF.

Molinié M., \& Leray, C. (2002)."Le voyage à l'étranger : un déplacement formateur". In Sabatier, C., Malewska, H. \& Tanon, F. (dir.). Identités, acculturation et altérité. Paris : L'Harmattan, pp. 230-238.

Murphy-Lejeune, E. (2005). L'étudiant européen voyageur, un nouvel étranger. Paris : Didier.

Pothier, M. (2003). Multimédias, dispositifs d'apprentissage et acquisition des langues. Paris : Ophrys. Disponible en ligne : http://edutice.archives-ouvertes.fr

Rosen, É. (2006). Le point sur le Cadre européen commun de référence pour les langues. Paris : Clé International.

Salengros-Iguenane, I. (2010a). "Français, francophones et non francophones dans une grande école : convergences et divergences". Actes du 38ème congrès de l'Uplegess « Enseignement des langues et cultures : comment former des acteurs responsables dans un monde sans frontières ?».pp. 59-66. Disponible en ligne : http://didatic.net/article.php3?id_article=229 Salengros-Iguenane, I. (2010b). Fle, Internet et approche culturelle - Une recherche à l'École nationale des ponts et chaussées. Thèse de sciences du langage. Université d'Aix - Marseille 1. Disponible en ligne : http://tel.archives-ouvertes.fr/tel-00556669/fr/

Scheepers, C. (2006). "L'écriture réflexive, entrée dans la culture". Molinié, M., (2006) (coord.). Bibliographie langagière et apprentissage plurilingue. Le français dans le monde - Recherches et applications, pp. 96-105.

Springer, C. (1996). La didactique des langues face aux défis de la formation des adultes. Paris : Ophrys. Tardif, J. (1998). Intégrer les nouvelles technologies de l'information. Quel cadre pédagogique? Paris : ESF.

Vézina, R. (2002). Portfolio sur support numérique. Québec : Ministère de l'Education du Québec. Direction des ressources numériques.

\section{NOTES}

1. D'après l'article de C. Scheepers (2006).

\section{RÉSUMÉS}

Nous nous intéressons aux difficultés culturelles des étudiants en mobilité à l'École des Ponts ParisTech, pour les aider, dans un module intégrant les TIC, à mieux s'adapter à la communauté française de l'école. Les apprenants ont produit un journal de bord, ce qui implique la mise en place d'un apprentissage par l'exploration et une prise de conscience, que l'on sera le «maître » 
de sa formation. Notre analyse montrera comment ils se sont investis dans la formation. Nous mettrons en valeur le cheminement personnel et une certaine relativisation culturelle. Nos résultats permettent de compléter les travaux antérieurs et montrent en quoi le journal de bord est un outil pertinent pour le public spécifique des grandes écoles

Our approach deals with both navigating the cultural difficulties incoming students to the École des Ponts ParisTech may experience, and designing and implementing a module using Internet. A central part of the module was journal writing by the students. It allows the learner to relate his/ her actual experience and thoughts. Our results complement former research and emphasize the fact that journals follow the learner's personal line of thought and reflect his/her process of cultural assimilation. This article focuses on the way learners have reacted to this task and how they put a lot of effort into doing the journal (self-assessment and learner's journal). This paper shows how a journal is a relevant tool for the specific public of a French graduate Engineering school.

\section{INDEX}

Keywords : adult education, autonomy, cultural approach, French as a Foreign Language, Internet, journal, knowledge construction, language learning methodology and cultures, personal learning path

Mots-clés : approche culturelle, autonomie, cheminement personnel, construction des connaissances, didactique des langues, FLE, formation d'adultes, Internet, journal de bord

\section{AUTEUR}

\section{ISABELLE SALENGROS-IGUENANE}

Isabelle Salengros-Iguenane travaille sur l'intégration d'Internet comme support pour la pédagogie en classe de français langue étrangère et sur l'interculturel, thèmes abordés dans son doctorat de didactique des langues. Elle est en poste à l'École Nationale des Ponts et Chaussées où elle poursuit ses recherches et enseigne la langue française.

Courriel : isabelle.salengros@enpc.fr

Adresse : École Nationale des Ponts et Chaussées, 6 et 8 avenue Blaise Pascal, Cité Descartes, Champs-sur-Marne, 77455 Marne-La-Vallée cedex 2. 